\title{
DIATOMEAS BENTÓNICAS DEL RÍO LOCUMBA, TACNA
}

\author{
Juan Franco León' \\ R E S U M E N \\ $r$ \\ RML
}

\begin{abstract}
Se dan a conocer los resullados del análisis de las muestras de diatomeas coleccionadas en cuato estaciomes delimitadas en el Río Locumba, Tarna. Lina colección de veinte muestras por estación, fueron lavadas por el método rápido convencional para lograr su identificación mediante un microscopio binocular con cámara folográfica incorporada y cámara lúcida para esquemas. Se han determinado treinta especies reportadas por otros autores y ocho géneros en los que predominan: Rhopalodia, Surirella, Synedra y Cyclotella.
\end{abstract}

\section{INTRODUCCION}

El material estudiado en este trabajo, en una primera etapa, constituye un primer reporte de Diatomeas de Ríos Costeros (R. Locumba), en el sur del Perú.

El cuerpo de agua analizada alberga alrededor de treinta especies, quedando aún muchas por describir. Al parecer su distribución está estrechamente relacionada con los factores físico químicos del medio que determinan su desarrollo.

La presencia de alta diversidad de Diatomeas en el Río Locumba, puede indicarnos referencias acerca de la calidad de agua en la cual viven y su estado de contaminación. Forman parte del inventario del catálogo de Diatomeas que se elabora actualmente en el Sur.

\section{MATERIAL Y MÉTODOS}

La colección de las muestras se hizo en forma periódica durante los meses de junio-setiembre de 1995. Las muestras fueron recogidas de la parte media y fondo del río, en forma directa, también se utilizó una red de fitoplancton; las muestras fueron depositadas en frascos de boca ancha y conservadas en una solución de formol al $5 \%$.

Referente al método de eliminación de la materia orgánica, se llevó a cabo mediante el

1. Biólogo. En colaboración de L. Cabana, P. Torres, G. Machaca y R Mariña procedimiento rápido o cruento, el cual consiste en cubrir el material lavado y contenido en una cápsula de porcelana con ácido sulfúrico concentrado comercial (procedimiento llevado a cabo bajo campana de gases). Sometido al calor (utilizando una llama moderada de mechero Bunsen o, en caso de no disponer de gas, mechero de alcohol) el color del material se torna negro después de hervir por unos 20 minutos. Al cabo de este tiempo se agrega $40 \mathrm{~cm}$ o más de ácido nítrico con ayuda de una pipeta larga en pequeñas cantidades y por los bordes de la cápsula, hasta que se produzca la decoloración del material, pasando del negro primitivo a la decoloración completa. En esta operación hay que tomar precauciones, pues la muestra líquida emite proyecciones al agregar ácido.

Al finalizar esta primera etapa, se retira la llama del mechero y se deja enfriar. El contenido frío se pasa a tubos de centrífuga donde se procede a efectuar los lavados, que deben extenderse hasta que desaparezcan los vestigios de ácido, usando agua destilada. Generalmente con seis operaciones, como las descritas, es suficiente para eliminar positivamente los restos de ácido, y evitar que este siga actuando y destruyendo los frúsculos de las diatomeas.

Una vez finalizada esta operación se debe guardar el material en recipientes bien cerrados, donde se agregan unas gotas de formol o una porción de timol para evitar los procesos de putrefacción.

Las muestras se analizaron realizando preparaciones en fresco para su observación directa, 
en microscopio compuesto binocular Karl Zeiss, que posee una cámara fotográfica incorporada. La identificación se realizó mediante el estudio morfológico por comparación y descripción de claves taxonómicas y realizando mediciones con ocular micrométrico.

\section{RESULTADOS}

Reseña taxonómica de Diatomeas del río Locumba, Tacna.

\section{División Bacillariophyta}

Orden Naviculales

Familia: Navicula muticopsis (Gran) Frena' Navicula mutica var. Peruviana (manguin) ${ }^{2}$ Navicula radiosa (Kutz) ${ }^{3}$

Navicula cuspidata(Kutz) ${ }^{4}$

Navicula gracilis (Hust) ${ }^{5}$

Navicula subtilisima (Cleve) ${ }^{6}$

Especie: Frustulia franguellii (Frang) ${ }^{7}$

Especie: Gyrosigma atenuatum (Ehrenbing) ${ }^{8}$

Especie:Pleurosigna (Quekett) ${ }^{9}$

Especie: Pinnulatia sp (Manguin) ${ }^{10}$

Especie: Stauroneis subtilis (Ehr.) ${ }^{11}$

Stauroneis anceps $(\mathrm{Ehr})^{12}$

Familia : Cymbellaceae

Especie:Cymbella sp $\quad(\text { Greg })^{13}$
Especie:Amphora ovalis (Kutz) ${ }^{14}$

Familia : Gomphonemaceae

Especie: Gomphonema sp (Kutz) ${ }^{15}$

Orden Surirellales

Familia: Surirellaceae

Especie: Surirella ovata(Kutz ${ }^{16}$

Surirella robusta(Ehr) ${ }^{17}$

Surirella delicatisima(Lewis) ${ }^{18}$

Orden Epithemiales.

Familia : Epothemiaceae

Especie:Ephitemia (Brebisson) ${ }^{19}$

Especie:Rhopalodia gibberula (Ehrrenb) muller ${ }^{20}$

Especie:Rhopalodia gibba (Ehrenb) muller ${ }^{21}$

Orden Nitzschiales

Familia: Nitzchiaceae

Especie: Nitzschia frustoloides (Gran) ${ }^{22}$

Nitzschia molesta (manguin) ${ }^{23}$

Nitzschia sp (Hassall) ${ }^{24}$

Orden Achnanthales

Familia: Achnanthaceae

Especie: Cocoveis placentuta (Ehreng) ${ }^{25}$

Orden Coscinodiseales

Familia: Thalassiosiraceae

Especie: Cyclotella meneghiniana (Kutzing) ${ }^{26}$

\section{HEMEROTECA CENTRAL UNIBG}

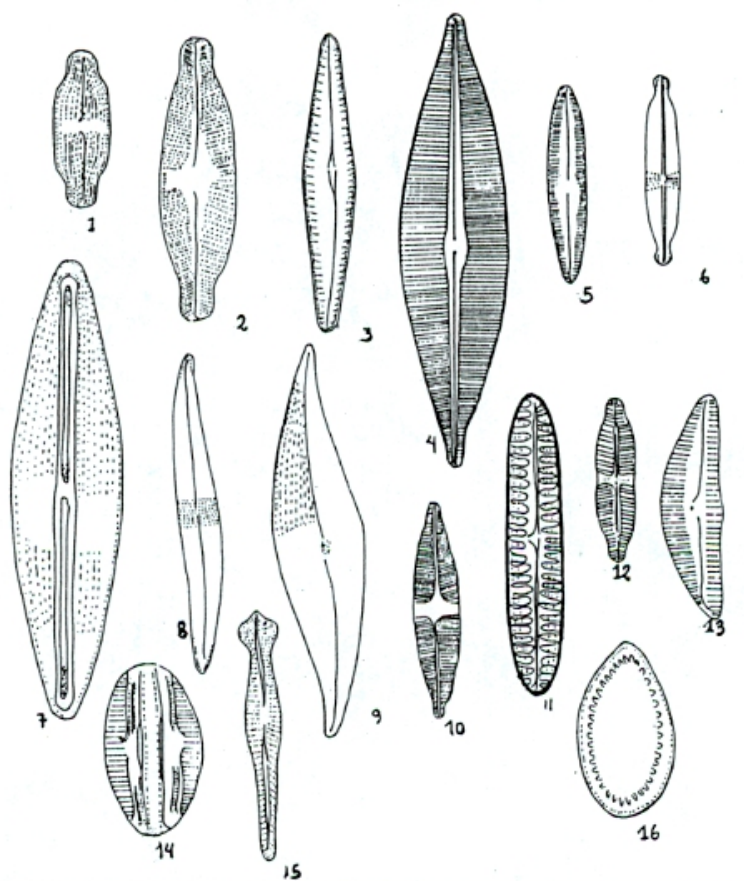




\section{DISCUSIÓN}

En el Río Locumba coexisten alrededor de treinta especies de Diatomeas agrupadas en siete órdenes. El papel principal dentro de esta comunidad plactónica está dado por las nitzschias, seguidas de las rhopalodias, grupo de micro algas extremadamente grande, con alta diversidad morfológica. Su distribución se reporta para la mayoria de aguas Continentales de la costa y sierra del Perú (Zelada, 1993).

Conforme a la clasificación realizada, la mayoria de ellas se ubicaron dentro de las muy comunes y raras, lo cual es normal en ambientes donde hay frecuencia de silicatos como elementos necesarios para su desarrollo. (Margolef 1988).

\section{REFERENCIAS BIBLIOGRÁFICAS}

CALLA, P. A. 1981. Identificación de Algas de Agua Dulce. Un nuevo Método para la identificación. Informe Presentado para obtener el Título Profesional. Arequipa-Perú.

FERNANDEZ, A. Y. AGUADO H. 1987. Botánica Criptogámica. Ed. U.N.T. Trujillo-Perú.

FRANCO, L.; DELGADO, V:: CASTILLO, D., LLOJA, L. Avances Preliminares del Estudio Sistemático de las Algas del Rio Caplina Revista Nueva Imagen - UNJBGTacna.
La composición de especies es coincidente con las obtenidas por otros investigadores, como la reportada por Chirinos (1994), para el río Totorani, Arequipa - 29 especies de Bacillariophytas, con dominancia de Rhopalodias y Pinnularias -, o la realizada por Delgado (1992) río Caplina-Tacna, donde predominan las diatomeas con abundantes synedras. Es decir, las especies reportadas para el río Locumba tienen amplia distribución, aún cuando falta completar la identificación de todas las especies que se presentan en el medio. Se debe mencionar también que algunas especies se comportan como indicadores de la contaminación de aguas.

GONZALES INFANTES. 1988. EI Plancton de las Aguas Continentales. Ed. Secretaria General de los Estados Americanos. Programa Regional de Desarrollo Cientifico y Tecnológico. Washington. USA. Fitoplancton de Aguas Polisaprobias (rio Torata-Moquegua). Escuela de Biologia-Microbiología.C.U. Tacna. Franco J. y Col.

ZUÑIGA A. 1988. Flora Criptogámica de Lima y Alrededores: Algas Continentales. Rev. Per. Biología. Lima-Perú.

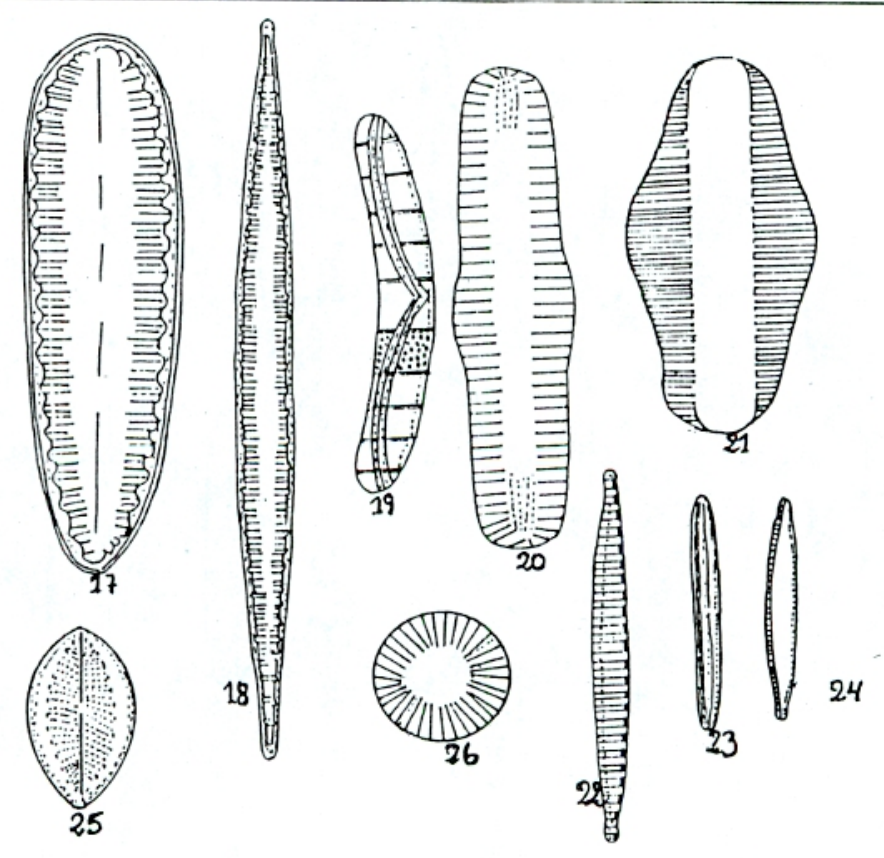

